\title{
Can Quantitative EEG Reliably Predict Deterioration from Delayed Cerebral Ischemia Secondary to Vasospasm?
}

\author{
J. Michael Schmidt $\cdot$ Jan Claassen
}

Published online: 1 March 2011

(C) Springer Science+Business Media, LLC 2011

Functional outcome after subarachnoid hemorrhage (SAH) has significantly improved over the past decades but secondary complications such as delayed cerebral ischemia (DCI) continue to take their toll [1]. In fact secondary complications are major determinants of outcome in neurocritical care [2]. Improving the detection of DCI has a high yield since it is frequent (approximately $25-40 \%$ of patients with $\mathrm{SAH}$ ), has a significant impact on outcome, and is potentially treatable when diagnosed in a timely fashion [3-6]. Treatment includes hypertensive hypervolemic therapy, nimodepine per mouth, intraarterial or intrathecal calcium channel blockers, and angioplasty. Most crucial for the effectiveness of these interventions is the timing of starting them.

To this date diagnosis of DCI at most institutions relies primarily on changes in the clinical examination and TCD, both of which are clearly limited and inadequate in a large portion of patients. Symptoms from DCI are extremely variable depending on the affected brain tissue and particularly in patients with high Hunt Hess grade, and therefore a poor baseline neurological exam, clinical changes may be extremely difficult to detect [3]. TCD, which are usually only obtained once a day, have been shown to have poor specificity and sensitivity for DCI [7-12].

This has led to the development of a number of ancillary diagnostic tests primarily to increase the sensitivity to

\author{
J. M. Schmidt · J. Claassen \\ Division of Critical Care Neurology, Department of Neurology, \\ Columbia University Medical Center, New York, NY 10032, \\ USA \\ J. Claassen $(\bowtie)$ \\ Milstein Hospital 8 Center, 177 Fort Washington Ave, \\ New York, NY 10032, USA \\ e-mail: jc1439@columbia.edu
}

detect DCI prompting angiographic confirmation. Most of these tests, such as CT angiography, MRI, or conventional angiography, have a poor temporal resolution or in other words cannot be obtained continuously. The ideal diagnostic test would have a very high sensitivity with reasonable specificity. It should be continuous and automatically alert physicians to initiate the appropriate next steps: first confirm the suspicion with a test that has a high specificity, and second initiate appropriate treatment if DCI secondary to vasospasm is confirmed.

Quantitative analysis of continuously recorded digital EEG data has the potential to offer many of these requirements. Prior studies have demonstrated that EEG changes can be seen with brain that is underperfused [13-15]. Actually, with decreasing perfusion sequential EEG changes are observed while brain tissue initially is in a state of reversible and then later irreversible brain injury [16]. A number of quantitative EEG parameters have been shown to correlate with DCI and vasospasm after SAH $[17,18]$ and may indicate this secondary complication up to 2 days prior to other tests [17]. However, all of these prior studies were conducted in an artificial study setting, which is one of the reasons that this technique is not widely used. What remains to be solved is a practical way to integrate this test into daily clinical practice.

Rathakrishnan and colleagues report in this issue of Neurocritical Care on a first attempt to integrate qEEG into the daily clinical routine of managing SAH patients in the Neuro ICU. They studied of a subgroup of 12 SAH patients deemed at high risk for DCI and found increasing accuracy when comparing predictions for clinical deterioration made purely on clinical data versus clinical data together with qEEG findings. Prediction of clinical deterioration improved from 40 to $67 \%$ with the use of qEEG data and clinical improvement from 8 to $50 \%$. In a small subset of 
these patients, EEG predicted clinical changes more than $24 \mathrm{~h}$ prior to changes of exam findings. Interestingly the qEEG parameter was promising in tracking treatment response and recurrence of DCI. While being very preliminary, this study is remarkable since it is the first to make an effort to determine the real-world practical value of qEEG monitoring in these patients.

It is unclear why the authors introduce a completely new qEEG parameter and do not compare this parameter to previously established EEG tools such as relative alpha variability [17] or alpha delta ratios. [18] Relative alpha variability scores in particular have quite a body of evidence studying their significance in other types of acute brain injury and predictive power compared to long-term functional outcome [19, 20]. Future studies will benefit from comparing the value of different qEEG parameters.

The true real-world test for this technique would be to determine the accuracy of predictions when paired up with qEEG triggered angiography. Many questions remain and the biggest concern is the specificity. A test with frequent false positive alarms will lead physicians to disregard the technique and potentially put the patient at increased risk from unnecessary imaging tests requiring in-hospital transportation, renal injury from iodine load, and increased radiation exposure. Very careful evaluation of the effects of hydrocephalus, medications, seizures, and ICP crises on qEEG variables are needed. Studies will have to compare all previously described qEEG parameters to identify the most accurate one. EEG data needs to be analyzed with and without preprocessing by automated artifact rejection algorithms. Expected changes from interventions need to be systematically evaluated.

The potential of utilizing continuously processed qEEG data for brain injured patients is tremendous, ideally leading to automated neurotelemetry in the future. These data will need to be incorporated with the vast amount of other physiologic monitoring data generated in the ICU setting. The greatest challenge for integrating these data into clinical practice is the danger of information overload which is a common occurrence for ICU physicians and nurses [2, 21]. Clinical informatics tools need to be developed to provide digestible data that would lead to management changes that potentially improve outcome. These data streams could continuously test preformed hypothesis such as "is there DCI?" and should incorporate quantified EEG parameters.

\section{References}

1. Komotar RJ, Zacharia BE, Valhora R, Mocco J, Connolly ES Jr. Advances in vasospasm treatment and prevention. J Neurol Sci. 2007;261:134-42.
2. Sorani MD, Hemphill JC 3rd, Morabito D, Rosenthal G, Manley GT. New approaches to physiological informatics in neurocritical care. Neurocrit Care. 2007;7:45-52.

3. Schmidt JM, Wartenberg KE, Fernandez A, et al. Frequency and clinical impact of asymptomatic cerebral infarction due to vasospasm after subarachnoid hemorrhage. J Neurosurg. 2008;109: 1052-9.

4. Rabinstein A, Pichelmann M, Friedman J, et al. Symptomatic vasospasm and outcomes following aneurysmal subarachnoid hemorrhage: a comparison between surgical repair and endovascular coil occlusion. J Neurosurg. 2003;98:319-25.

5. Wartenberg KE, Schmidt JM, Claassen J, et al. Impact of medical complications on outcome after subarachnoid hemorrhage. Crit Care Med. 2006;34:617-23.

6. Treggiari-Venzi MM, Suter PM, Romand JA. Review of medical prevention of vasospasm after aneurysmal subarachnoid hemorrhage: a problem of neurointensive care. Neurosurgery. 2001;48: 249-61. discussion 61-2.

7. Carrera E, Schmidt JM, Oddo M, et al. Transcranial Doppler for predicting delayed cerebral ischemia after subarachnoid hemorrhage. Neurosurgery. 2009;65:316-23. discussion 23-4.

8. Lysakowski C, Walder B, Costanza MC, Tramer MR. Transcranial Doppler versus angiography in patients with vasospasm due to a ruptured cerebral aneurysm: a systematic review. Stroke. 2001;32:2292-8.

9. Sekhar LN, Wechsler LR, Yonas H, Luyckx K, Obrist W. Value of transcranial Doppler examination in the diagnosis of cerebral vasospasm after subarachnoid hemorrhage. Neurosurgery. 1988; 22:813-21.

10. Harders AG, Gilsbach JM. Time course of blood velocity changes related to vasospasm in the circle of Willis measured by transcranial Doppler ultrasound. J Neurosurg. 1987;66:718-28.

11. Naval NS, Thomas CE, Urrutia VC. Relative changes in flow velocities in vasospasm after subarachnoid hemorrhage: a transcranial Doppler study. Neurocrit Care. 2005;2:133-40.

12. Grosset DG, Straiton J, McDonald I, Cockburn M, Bullock R. Use of transcranial Doppler sonography to predict development of a delayed ischemic deficit after subarachnoid hemorrhage. J Neurosurg. 1993;78:183-7.

13. van Putten MJ, Tavy DL. Continuous quantitative EEG monitoring in hemispheric stroke patients using the brain symmetry index. Stroke. 2004;35:2489-92.

14. Sharbrough FW, Messick JM Jr, Sundt TM Jr. Correlation of continuous electroencephalograms with cerebral blood flow measurements during carotid endarterectomy. Stroke. 1973;4:67483.

15. Finnigan SP, Rose SE, Walsh M, et al. Correlation of quantitative EEG in acute ischemic stroke with 30-day NIHSS score: comparison with diffusion and perfusion MRI. Stroke. 2004;35:899903.

16. Jordan KG. Emergency EEG and continuous EEG monitoring in acute ischemic stroke. J Clin Neurophysiol. 2004;21:341-52.

17. Vespa PM, Nuwer MR, Juhasz C, et al. Early detection of vasospasm after acute subarachnoid hemorrhage using continuous EEG ICU monitoring. Electroencephalogr Clin Neurophysiol. 1997;103:607-15.

18. Claassen J, Hirsch LJ, Kreiter KT, et al. Quantitative continuous EEG for detecting delayed cerebral ischemia in patients with poor-grade subarachnoid hemorrhage. Clin Neurophysiol. 2004; 115:2699-710.

19. Vespa PM, Boscardin WJ, Hovda DA, et al. Early and persistent impaired percent alpha variability on continuous electroencephalography monitoring as predictive of poor outcome after traumatic brain injury. J Neurosurg. 2002;97:84-92.

20. Hebb MO, McArthur DL, Alger J, et al. Impaired percent alpha variability on continuous electroencephalography is associated 
with thalamic injury and predicts poor long-term outcome after human traumatic brain injury. J Neurotrauma. 2007;24: 579-90.
21. Morris G, Gardner R. Computer applications. In: Hall J, Schmidt G, Wood L, editors. Principles of critical care. New York: McGraw-Hill; 1992. p. 500-14. 